\title{
Influence of hydrodynamic dispersion and nonequilibrium sorption on the prediction of pesticide concentrations in lysimetric leachate
}

\author{
Victoria Kolupaeva ${ }^{1, *}$, and Anna Kokoreva ${ }^{2}$ \\ 1 Federal State Funded Research Institution All-Russian Research Institute of Phytopathology, \\ 143050, 5, Institute Str., Bolshie Vyazemy, Moscow region, Russian Federation \\ 2 Moscow State University named after M.V. Lomonosov, 119991, 1, Leninskie gory, Moscow, \\ Russian Federation
}

\begin{abstract}
The article discusses issues related to the development of pesticide modeling in the environment and its use in order to regulate the circulation of pesticides. As the study of the literature and the author's experience in using mathematical models shows, the main problem and source of errors in modeling is the quality of the input data for the soil block of the model, as well as those characterizing the properties of the pesticide. Using the results of a carefully conducted lysimetric experiment to study the migration of cyantraniliprole outside the soil profile, as well as auxiliary studies, the influence of hydrodynamic dispersion on the behavior of the insecticide and the results of modeling is shown. The parameters of its non-equilibrium sorption by the soil were calculated according to the data of the laboratory experiment investigating the dynamics of decomposition of cyantraniliprole. The article confirms the need to take these processes into account when modeling the transport of pesticides.
\end{abstract}

\section{Introduction}

Pesticide contamination of groundwater is a concern as it affects virtually all agricultural regions of the world. Unlike other pollutants such as pharmaceuticals, pesticides are deliberately introduced into the soil by humans, and this process can and shall be regulated to prevent or minimize adverse environmental impacts. To this end, before accessing the market, the active ingredients of pesticides and their formulations are thoroughly studied in order to assess the risk of their use. One of the steps in this process is the assessment of the risk of migration of pesticides to groundwater, which is carried out using pesticide fate model.

Mathematical models describing the behavior of pesticides in environmental objects appeared in the mid-70s and were later used in a number of European countries when registering pesticides, for example, in Germany and the Netherlands. With the entry into force of the EU directive 91/414/ EEC [1] in 1993, the modeling results - the so-called PEC (Predicted Environmental Concentration) were added to the list of required pesticide

\footnotetext{
*Corresponding author: v.kolupaeva@vniif.ru
} 
information along with experimental data and began to play an important role in the process of making decisions on the inclusion of a pesticide in the list of approved active ingredients of preparations. In the Russian Federation, the results of mathematical modeling of migration of a. pesticides from soil to groundwater since 2007 have also been included in the pesticide data sheet and in the decision tree for assessing the risk of groundwater contamination. But, despite the fact that the experience of using models for regulatory purposes has almost 30 years, nevertheless, the accuracy of describing the processes of migration of pesticides in soil by models remains low. Poor predictive ability of absolute pesticide concentrations does not make the model unsuitable for the selection of active substances that can adversely affect groundwater, or for their correct ranking in terms of the risk of leaching.

Many studies have been undertaken in order to evaluate the accuracy of the models by comparing the forecast with the experimental data. In general, it was found that the prediction of the distribution of pesticides along the profile is more accurate than the prediction of the concentration of pesticides in the leachate, and the models based on the Richards equation (PEARL, MACRO) are superior to the models of the capacitive (balance - PRZM, PELMO) type [2-4]. Unsatisfactory forecast accuracy can be caused either by errors of the model itself (incorrect description of the processes occurring) or parameterization errors (inaccurate determination of the input parameters of the model). To reduce errors of the first type, the existing models are being improved in describing nonequilibrium phenomena in the description of transport, transformation and sorption processes, such as flow through macropores, kinetic adsorption, and time-dependent decomposition. Nevertheless, many authors believe [2] that the input data have a greater influence on the simulation results than the properties of the model itself. The improvement in model performance after "physically justified calibration" also indicates that the poor quality of the forecast is due to inaccuracies in parameterization.

Calibration of models is carried out in stages. At the first stage, the accuracy is assessed and, if necessary, the adjustment of the water block is carried out: the experimental and predicted distributions of moisture in the soil and water flow at the lower boundary are compared. In balance models, as a rule, the parameters of evapotranspiration are adjusted, in models based on the convective-dispersion equation, the parameters of the equations describing the basic hydro-physical characteristics and the function of moisture conductivity [5-8]. Adjustment according to the moisture profile does not always give good indicators of accuracy for the flow, and vice versa. Burau [7] believes that it is necessary to adjust, first of all, the parameters calculated using the pedotransfer function, and only then those obtained experimentally, and one shall strive to minimize the number of optimized variables. Most researchers agree that the models describe satisfactorily the moisture profile, water balance and water percolation [5-8] after adjustment. In a number of studies, the values of the parameter ME (model efficiency, Nash-Sutcliffe coefficient) of the moisture forecast vary from 0.21 to 0.9 , and the scaled root mean square error (SRMSE) from 0.05 to 0.11 [6-9]. The accuracy of simulation of water percolation, as a rule, turns out to be lower and requires adjustment [5-9].

The situation with modeling the migration of pesticides is more complicated. Even after fitting the water and pesticide parameters, it is not always possible to achieve a satisfactory adjustment $[6,8,9]$, and calibration with an acceptable accuracy along the pesticide distribution profile rarely leads to successful predictions of pesticide concentrations in the leachate [6]. There may be several reasons for this. First, the main characteristics that determine the fate of the pesticide, the half-life DT50, and the sorption constant $\mathrm{Kd}$ are determined in laboratory experiments and cannot always be extrapolated to field conditions [10]. The second reason may be the phenomenon of preferential flows, widely known and described in the literature [11]. At present, efforts are being made to include a computation 
unit describing the movement of water and solutions with preferential flowsin all pesticide fate models [12]. However, to describe the macropore transport by the model, additional input data are required, which are difficult to measure in a direct experiment. Third, the model contains parameters whose values are entered by default: the dispersion length (Ldis), which describes the hydrodynamic dispersion, and the kdes and fNE parameters, which characterize the nonequilibrium sorption of pesticides. By default, it is recommended to use the mixing dispersion length value equal to $5 \mathrm{~cm}$, however, the values determined in laboratory and field experiments for a number of soils were much higher - up to $40.3 \mathrm{~cm}$ in soil monoliths $30 \mathrm{~cm}$ high $[12,13]$. The size of the dispersion length affects the rate of migration of pesticides in the soil, since with its increasing, the front of movement of the substance is blurred, and the pesticide is detected earlier at the lower boundary.

Nonequilibrium sorption is another process that significantly affects the transport of substances in the soil. Non-equilibrium sorption is understood as a magnification in the degree of binding of pesticides to the soil as the interaction time between them increases, while the bioavailability of the pesticide and its ability to migrate decrease. There are many names for this phenomenon, such as 'aged sorption', 'time-dependent sorption', 'increase in sorption over time', 'kinetic sorption' and 'non-equilibrium sorption'. The parameters of nonequilibrium sorption can be determined in a laboratory experiment. The recommended default values for kdes and fNE are 0.01 and 0.3 , respectively [14].

The aim of this study was to study the effect of hydrodynamic dispersion and kinetic sorption on the migration of the pesticide cyantraniliprole. The first task was to calibrate the PEARL model by the dispersion length using the data on the concentration of the pesticide in the lysimetric water, or, in other words, using the inverse modeling method to optimize the "dispersion length" parameter according to the lysimetric experiment and compare its values with the values obtained in laboratory filtration study and in field experiments with coloring solution. The second task was to calculate the parameters of the nonequilibrium sorption of cyantraniliprole using the data of the laboratory incubation experiment and to assess its effect on the migration of this insecticide in the soil.

\section{Methods}

The study of the behavior of the insecticide cyantraniliprole was carried out in the Large lysimeters of the Moscow State University. The experimental procedure, as well as the properties of soil and insecticide, are described by Kolupaeva et al. [15]. The properties of the PEARL model and the input parameters to it are given in the articles by Kolupaeva et al. [nine].

The differences between the experimental and calculated values were estimated using statistical parameters: the scaled root-mean-square error (Equation 1) and the model efficiency indicator - the Nash-Sutcliffe coefficient (Equation 2):

$$
\begin{gathered}
\text { SRMSE }=\frac{1}{\bar{o}} \sqrt{\frac{1}{n}} \sum_{i=1}^{n}\left(O_{i}-P_{i}\right)^{2} \\
M E=\frac{\sum_{i=1}^{n}\left(O_{i}-\bar{O}\right)^{2}-\sum_{i=1}^{n}\left(P_{i}-\bar{O}\right)^{2}}{\sum_{i=1}^{n}\left(O_{i}-\bar{O}\right)}
\end{gathered}
$$

where Oi and Pi are observed and calculated values, respectively, $\bar{O}$ is the average of the experimental values, $\mathrm{n}$ is the number of measurements. SRMSE shows the scatter around the mean of the measurements, and the ME indicates whether the model describes the data 
better than just the mean. The optimal values for SRMSE and ME are zero and one, respectively.

\section{Results}

Studies of the behavior of cyantraniliprole were carried out in lysimeters at Moscow State University in 2015-2018. The pesticide was applied at a dose of $4 \mathrm{~kg} / \mathrm{ha}$. The model was parameterized using the data of Kolupaeva et al. [6, 9]. During the simulation, the conditions were chosen at the lower boundary - a lysimeter. Information on the half-life (DT50 49.9 days) and the sorption constant (Kd $5.8 \mathrm{ml} / \mathrm{g}$ ) of cyantraniliprole were obtained in laboratory experiments. $[16,17]$. The adjustment was carried out first by the parameter "dispersion length" (Ldis) at fixed values of DT50 and Kd, then by two parameters - the dispersion length and the sorption constant, and by all three parameters, using the value of the Nash-Sutcliffe coefficient as a criterion for the forecast accuracy.

The calculated and experimental concentrations of cyantraniliprole in the percolate after adjustment for one fitted parameter - the dispersion length are shown in Figure 1. The best fit of the calculated and experimental concentrations was obtained at a dispersion length of $40 \mathrm{~cm}$. As can be seen in the figure, after calibrating and obtaining satisfactory values of the ME coefficient (Table 1), the model underestimates experimental data for the first 6 months after the pesticide was applied: the substance in the experiment was detected in June 2015, while, according to the simulation, was appeared in leachate in February 2016. In addition, in two samples in June 2015, the concentration of the insecticide reached very high values, after their discarding, the prediction accuracy increased. The calculation for the recommended dispersion length value of $5 \mathrm{~cm}$ is not given, since in this case the pesticide concentration in water was zero.

When calibrating the model for two (dispersion length and sorption constant) and three (dispersion length, sorption constant, and half-life) parameters, it was not possible to significantly improve the prediction accuracy.

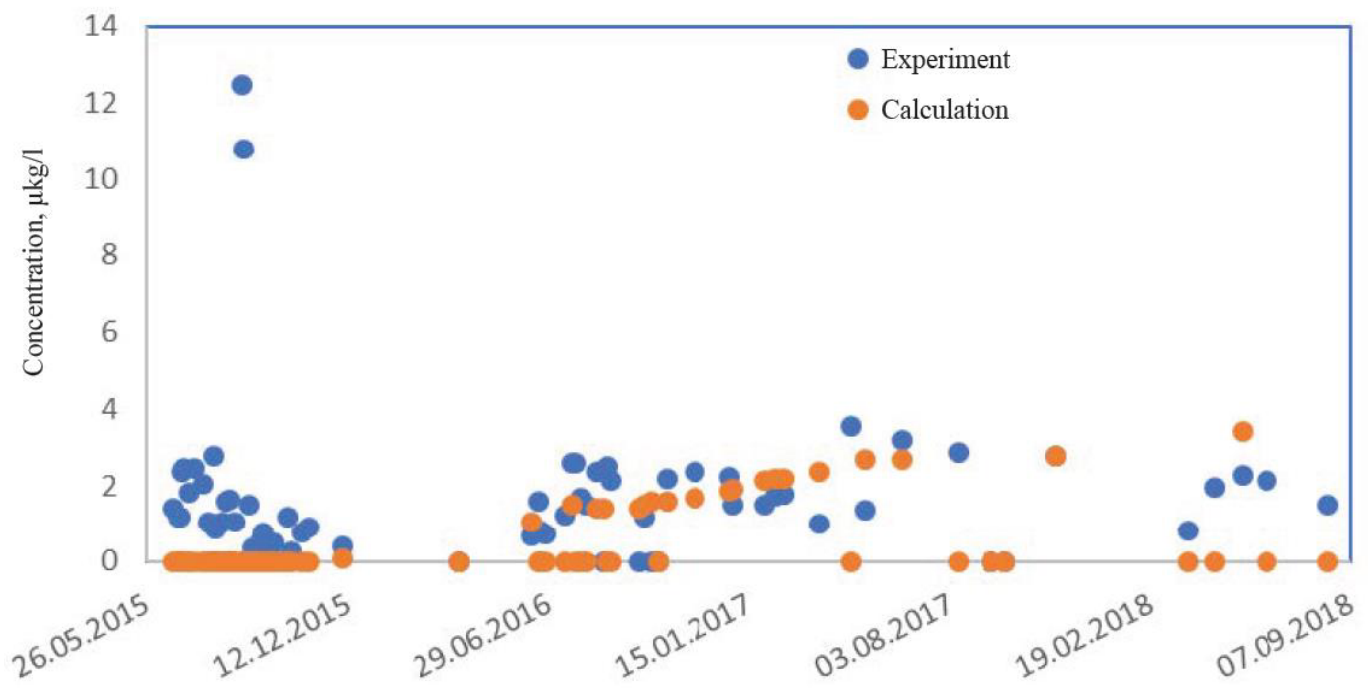

Fig. 1. Calculated and experimental concentrations of cyantraniliprole in the leachate. 
Table 1. Statistical parameters of simulation accuracy.

\begin{tabular}{|l|c|c|c|c|}
\hline \multirow{2}{*}{ Parameter } & \multicolumn{2}{c|}{ No rejection } & \multicolumn{2}{c|}{ With rejection } \\
\cline { 2 - 5 } & Experiment & Simulation & Experiment & Simulation \\
\hline Average & 1.61 & 0.52 & 1.33 & 0.53 \\
\hline Standard deviation & 1.92 & 0.92 & 0.92 & 0.93 \\
\hline $\mathrm{n}$ & 74 & 74 & 72 & 72 \\
\hline ME & & -0.51 & & 0.50 \\
\hline SRMSE & & 1.45 & & 1.02 \\
\hline
\end{tabular}

The presentation of the data, as shown in Figure 2, where the experimental values were indicated on the abscissa and the calculated values on the ordinate indicates that there is a large number of zero predicted concentration values in the presence of the pesticide in the corresponding samples. Perhaps this was due to the fact that in the experiment, water samples were taken 1-2 times a week and the concentrations were averaged over several days.



Fig. 2. Calculated and experimental concentrations of cyantraniliprole in the leachate.

The second process, which was considered in our paper, is non-equilibrium sorption, which is due to the intensification of the interaction of the soil with the pesticide over time and leads to the loss of a part of the mobility and bioavailability of the substance remaining in the soil. If this process is not taken into account in modeling, it can lead to overestimation of pesticide migration. It is the fact that a part of the pesticide passes into a non-equilibrium bound state that leads to a decrease in the rate of degradation of pesticides in the soil. This phenomenon is called two-stage decomposition. In order to determine the parameters of nonequilibrium sorption, an incubation laboratory experiment is usually carried out and the residual amounts of the pesticide are determined by extraction with an organic solvent and water extraction. However, there are also recommendations for finding the parameters of nonequilibrium sorption based on the results of a simple incubation experiment (extraction with only an organic solvent) using a simple calculation procedure from the parameters of the biexponential degradation equation [18]. Table 1 shows the obtained values of the parameters of the two-stage degradation of cyantraniliprole - $g, \lambda 1$, and $\lambda 2$, the parameters of nonequilibrium sorption - kdes (the rate of transition of a substance into a nonequilibrium phase), DT50 is the half-life in the equilibrium phase, fNE is the ratio of the sorption constant in the nonequilibrium and equilibrium phases. The resulting desorption constant kdes was close to the recommended values, while the fNE of 
3, 37 was much higher. This was consistent with the data of other authors that the parameters obtained as a result of such calculations overestimate the contribution of nonequilibrium sorption [19]. Using the PEARL model, the average long-term values of cyantraniliprole in the leachate were calculated for the variant without taking into account the nonequilibrium sorption, which turned up to be almost 50 times higher than taking into account this phenomenon according to the calculated data and 10 times higher according to the recommended parameters (Table 2).

Table 2. Parameters of the biexponential degradation and nonequilibrium sorption of cyantraniliprole, predicted concentrations in the leachate. Statistical characteristics of accuracy.

\begin{tabular}{|c|c|c|c|c|c|c|c|c|c|c|}
\hline $\begin{array}{l}g \\
(-)\end{array}$ & $\begin{array}{c}\lambda_{1} \\
\text { (day } \\
1 \text { ) }\end{array}$ & $\begin{array}{c}\lambda_{2} \\
\left(\text { day }^{-1}\right)\end{array}$ & $\begin{array}{c}\mathbf{k}_{\text {des }} \\
\left(\text { day }^{-1}\right)\end{array}$ & $\begin{array}{c}k_{t} \\
\left(\text { day }^{-1}\right)\end{array}$ & $\begin{array}{c}\text { DT } \\
50 \\
\text { (da } \\
\text { ys) }\end{array}$ & $\begin{array}{l}\Phi \\
(-)\end{array}$ & $\begin{array}{l}\mathbf{f}_{\mathrm{NE}} \\
(-)\end{array}$ & $\begin{array}{c}S_{g v} \\
\left(\mu g L^{-1}\right)\end{array}$ & SRMSE & ME \\
\hline 0.4 & $\begin{array}{c}0.07 \\
25 \\
\end{array}$ & 0.0047 & 0.0107 & 0.0318 & 21.8 & 3.24 & 3.37 & 0.04 & 0.03 & 0.99 \\
\hline \multicolumn{3}{|c|}{ Default } & 0.01 & & 49.9 & & 0.3 & 0.41 & & \\
\hline $\begin{array}{c}1.0 \\
*\end{array}$ & $\begin{array}{c}0.01 \\
39\end{array}$ & 0.0000 & 0.0000 & 0.0139 & 49.9 & - & 1 & 2.49 & 0.13 & 0.89 \\
\hline
\end{tabular}

* - calculation according to the first-order equation

Figure 3 shows again the experimental data on the concentrations of cyantraniliprole in the lysimetric water and two cases of calculation with optimized dispersion lengths $(40 \mathrm{~cm})$ - without and taking into account nonequilibrium sorption (default parameters, $\mathrm{k}_{\mathrm{des}}=0.01$ day- $1, f_{\mathrm{NE}}=0.3$ ). The figure shows that nonequilibrium sorption significantly affects the migration of the pesticide and leads to a decrease in the calculated concentrations. The case with the sorption parameters calculated according to the biexponential equation is not shown in the figure, since with such parameters cyantraniliprole in water was not predicted.

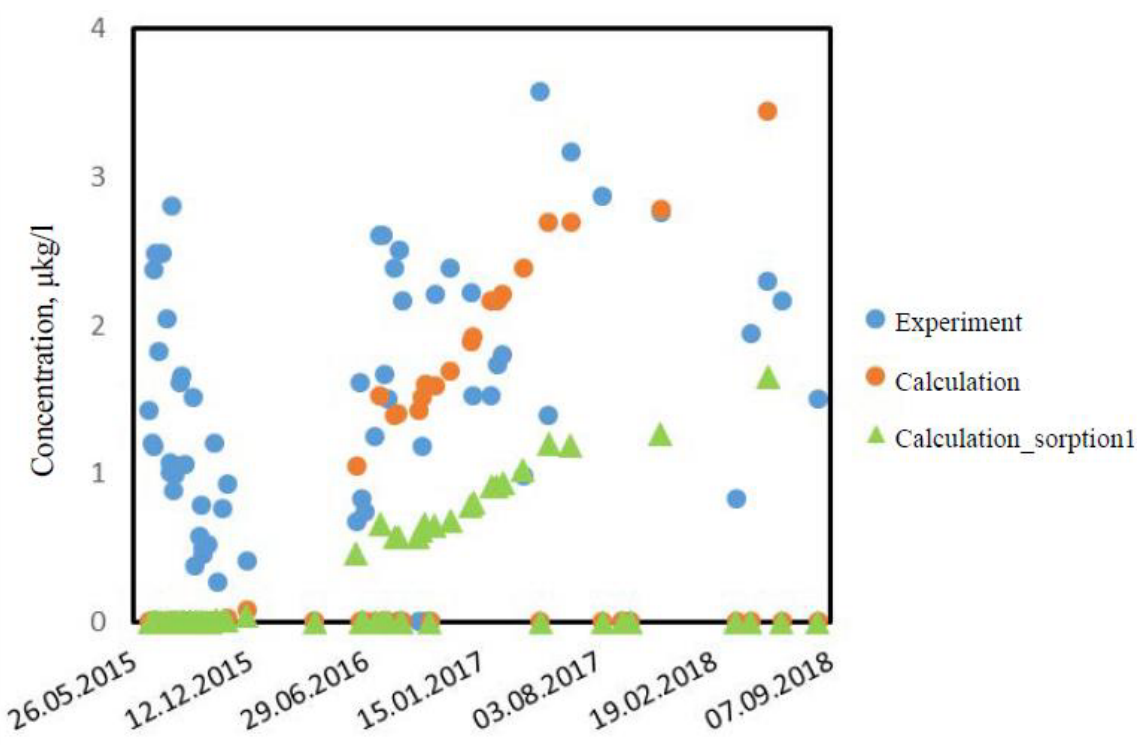

Fig.3. Concentrations of cyantraniliprole in the lysimetric water. 


\section{Discussion}

Considering the complexity of predicting the concentrations of pesticides in the leachate, as well as the large number of processes affecting migration, it can be assumed that, as a result of the optimization of the "dispersion length" parameter based on the data on the concentrations of cyantraniliprole in the lysimetric water, an acceptable prediction accuracy was achieved. It shall also be noted that the selected value of the dispersion length was $40 \mathrm{~cm}$, which is significantly higher than the one suggested by the model developers for use by default, but equal to the maximum value of this parameter obtained in a laboratory experiment in columns $30 \mathrm{~cm}$ long [13]. In this case, we observed the movement of the pesticide with predominant migration routes and the inability of the chromatographic type model to take this process into account. Optimization of the "dispersion length" parameter makes it possible to increase the adequacy of the pesticide transport model description.

Nonequilibrium sorption also significantly affected the transport of dissolved substances in the soil. Currently, in assessing the risk of pesticide migration, it is assumed that the ratio between sorbed and dissolved pesticide is established immediately after contact with the soil and that sorption is completely reversible ("sorption equilibrium"). This means that the strength of sorption is constant over time. However, it is well known that sorption is not really a simple instantaneous equilibrium process. The distribution coefficients between the solid and liquid phases during desorption in laboratory studies often exceed the adsorption coefficients (sorption hysteresis), which suggests that adsorption is reversible only partially within the time scale of the experiment. Equilibrium for many compounds is reached only after a few days, months, or even years, and the distribution coefficient can increase several times during this time [20]. In our study, the parameters of nonequilibrium sorption were calculated from the biexponential equation. The obtained fNE value - the ratio of the sorption constant in the nonequilibrium and equilibrium phases - is 10 times higher than the one offered for use by default. It can be assumed that the slowdown in the rate of degradation of the pesticide was due to a number of processes, one of which was an increase in the degree of binding of the pesticide to the soil, which was why the fNE value determined in this experiment was overestimated.

Therefore, from all of the above, it can be seen that multidirectional processes were observed in the soil - the movement of dissolved compounds with preferential flows, which we tried to simulate by optimizing the dispersion length and which accelerates the transport of the pesticide into lysimetric waters, and non-equilibrium sorption, which fixed the pesticide in the soil matrix, preventing its decomposition and migration. The accumulation of experimental data on the behavior of pesticides in lysimeters and parameters describing the nonequilibrium sorption and movement of substances along the preferential migration routes will contribute to the refinement of the input parameters to the models and lead to an increase in the adequacy of simulation, as well as to a more accurate understanding of the processes occurring with the pesticide.

\section{Conclusion}

The PEARL model was adjusted according to the data on the concentrations of cyantraniliprolee in the leachate by optimizing the dispersion length value. An ME value of 0.5 indicated acceptable calculation accuracy. The optimized dispersion length was $40 \mathrm{~cm}$.

The parameters of the nonequilibrium sorption of cyantraniliprole were calculated according to the data of the incubation experiment: $\mathrm{k}_{\mathrm{des}}=0.0107, \mathrm{f}_{\mathrm{NE}}=3.37$. An assessment of the influence of nonequilibrium sorption on the migration of an insecticide outside the soil profile was carried out. 
The work was performed within the framework of the State Assignment of the Federal State Funded Research Institution All-Russian Research Institute of Phytopathology, topic 0598-2019-0005.

\section{References}

1. EEC Council directive $91 / 414$ of $15 / 7 / 91$ concerning the placing of plant protection products on the market, Official Journal of the European Communities, 34 (1991)

2. M. Vanclooster, J.J.T.I. Boesten, Agricultural Water Management, 44 (2000)

3. J. A. Garratt, E. Capri, M. Trevisan, G. Errera, R. M. Wilkins, Italy Pest Manag Sci., 59, 1 (2003)

4. M. Vanclooster, J.D. Piñeros Garcet, J.J.T.I. Boesten, Université catholique de Louvain (2003)

5. K.V. Shishkin, A.A. Belik, A.A. Kokoreva, Z.S. Yezhelev, Byulleten' Pochvennogo instituta imeni V.V. Dokuchayeva, 99 (2019)

6. V.N. Kolupaeva, V.S. Gorbatov, A.A. Kokoreva, Environmental Fate and Ecological Effects of Pesticides (2010)

7. F. Bouraoui, Environmental Modelling \& Software, 22 (2007)

8. R.P. Scorza Junior, J. J. T. I. Boesten, Pest Manag Sci., 61 (2005)

9. V. N. Kolupayeva, V. S. Gorbatov, E.V. Shein, A. A. Leonova, Pochvovedeniye, 6 (2007)

10. P. J. Van den Brink, C. D. Brown, I. G. Dubus, Ecological Modelling, 191 (2006)

11. E.V. Shein, A.A. Belik, A.A. Kokoreva, V.N. Kolupaeva, Eurasian Soil Science, 51, 7 (2018)

12. A. Tiktak, R.F.A. Hendriks, J.J.T.I. Boesten, Pesticide Manag. Sci., 68 (2011)

13. Ye. V. Shein, A. A. Kokoreva, V. N. Kolupayeva, A. A. Belik, P. A. Pletenev, Agrokhimicheskiy vestnik, 6 (2016)

14. Report of the FOCUS ground water work group, EC Document Reference Sanco, 3 (2014)

15. V.N. Kolupaeva, A.A. Kokoreva, A.A. Belik, P.A. Pletenev, Open Agriculture, 4, 1 (2019)

16. V. Kolupaeva, I. Nyukhina, A. Belik, E3S Web of Conferences, 169 (2020)

17. V.N. Kolupayeva, V.S. Gorbatov, I.V. Nyukhina, Vestnik NGAU (Novosibirskiy gosudarstvennyy agrarnyy universitet), 39, 2 (2016)

18. Report of the FOCUS Work Group on Degradation Kinetics, EC Document Reference Sanco, 2 (2006)

19. M. X. Huang, American Chemical Society (Washington, 2014)

20. S. Beulke, W. van Beinum, L. Suddaby, Integrated Environmental Assessment and Management, 11, 2 (2015) 